The Astrophysical Journal, 617:480-489, 2004 December 10

(C) 2004. The American Astronomical Society. All rights reserved. Printed in U.S.A.

\title{
PULSAR WIND NEBULAE AND THE X-RAY EMISSION OF NONACCRETING NEUTRON STARS
}

\author{
K. S. Cheng, ${ }^{1}$ Ronald E. TaAm, ${ }^{2}$ and W. Wang ${ }^{1}$ \\ Received 2004 May 26; accepted 2004 August 24
}

\begin{abstract}
The general properties of the nonthermal nonpulsed X-ray emission of rotation-powered pulsars are investigated in the context of a pulsar wind nebula model. An examination of the observed X-ray emission from a sample of 23 pulsars in the energy range between 2 and $10 \mathrm{keV}$ reveals that the relation of X-ray luminosity, $L_{\mathrm{X}}$, to the pulsar spin-down power, $\dot{E}$, is steeper for the nonpulsed component than for the pulsed component. Specifically, $L_{\mathrm{X}}^{\text {npul }} \propto \dot{E}^{1.4 \pm 0.1}$ for the nonpulsed component, whereas $L_{\mathrm{X}}^{\text {pul }} \propto \dot{E}^{1.2 \pm 0.08}$ for the pulsed component. The former relation is consistent with emission from a pulsar wind nebula model in which $L_{\mathrm{X}}^{\mathrm{npul}} \propto \dot{E}^{p / 2}$, where $p$ is the powerlaw index of the electron energy distribution. The relation for the pulsed component, on the other hand, is consistent with a magnetospheric emission model. In addition, the photon spectral index, $\Gamma$, was found to be correlated with the conversion efficiency of spin-down power into nonpulsed X-ray emission, with greater efficiencies for $\Gamma \sim 2-2.5$ than for $\Gamma \sim 1.5-2$. Such a relation can be naturally understood within the framework of a pulsar wind nebula model, with the former relation corresponding to the emission of X-rays in the fast-cooling regime and the latter relation corresponding to emission in the slow-cooling regime. The X-ray properties of pulsar wind nebulae are sensitive to the physical conditions (e.g., the density and magnetic field) of the interstellar medium, which can lead to important differences between the X-ray emission characteristics (luminosity, photon spectral index, and emission morphology) of pulsars in various environments. Such wind nebulae can contribute to the nonthermal symmetric emission morphology (pointlike) and elongated emission morphology (tail-like) from sources similar to Geminga and PSR B1757-24.
\end{abstract}

Subject headings: pulsars: general — radiation mechanisms: nonthermal — stars: neutron — X-rays: stars

\section{INTRODUCTION}

The study of the emission characteristics from rotationpowered pulsars has been a subject of long-standing interest. Our knowledge of the fundamental properties (e.g., mass, spin, and magnetic field) of the underlying neutron star stems from detailed spectral and timing investigations. To facilitate an understanding of the mechanism by which the loss of rotational energy is converted into high-energy radiation, many observational and theoretical studies have sought to determine the relationship between the X-ray luminosity, $L_{\mathrm{X}}$, and the rate of rotational energy loss or spin-down power, $\dot{E}$. Indeed, a correlation of the form $L_{\mathrm{X}} \propto \dot{E}^{1.39}$ was found in Einstein data by Seward \& Wang (1988). Subsequent studies using a larger sample of pulsars led to a relation of the form $L_{\mathrm{X}} \propto \dot{E}$ based on ROSAT data (see Becker \& Trümper 1997) and $L_{\mathrm{X}} \propto \dot{E}^{1.5}$ based on $A S C A$ data (see Saito 1998). Recently, a reanalysis of 39 pulsars on the basis of data obtained from several X-ray satellites by Possenti et al. (2002) led to an intermediate relation, $L_{\mathrm{X}} \propto \dot{E}^{1.34}$, similar to that of Seward \& Wang (1988).

While the deduced existence of a correlation between $L_{X}$ and $\dot{E}$ suggests that the observed X-rays are produced by a process that taps the rotational energy of the neutron star, a detailed description of the mechanism remains elusive. This is, in part, a result of the fact that the data from different satellites are obtained in different energy ranges. The results can be affected by, for example, interstellar absorption, especially for those pulsars studied in the soft X-ray regime of ROSAT $(0.1-2.4 \mathrm{keV})$. In addition, the total X-ray luminosity is composed of con-

\footnotetext{
1 Department of Physics, University of Hong Kong, Pokfulam Road, Hong Kong.

2 Department of Physics and Astronomy, Northwestern University, 2145 Sheridan Road, Evanston, IL 60208.
}

tributions from both the pulsed and nonpulsed components, and these components are likely to reflect physical conditions in diverse spatial environments.

The X-rays radiated by rotation-powered pulsars could include contributions from five distinct components with different origins:

1. Nonthermal nonpulsed diffuse radiation can be emitted from a pulsar wind nebula. The radiation is discussed in $\S 2$, and we suggest that this emission component makes the major contribution to the nonpulsed X-ray radiation in the $A S C A$ observations of pulsars.

2. Nonthermal nonpulsed radiation from the pulsar magnetosphere could be important, as suggested by Becker et al. (2004), if the angular resolution of the X-ray detector is sufficiently high to separate this contribution from that of the nebula. Although such a component may be present in the $A S C A$ data that we use, owing to its low angular resolution it is not likely to dominate the contribution from the pulsar wind nebula. For example, Tennant et al. (2001) have found a nonthermal nonpulsed component from the Crab pulsar by using the HighResolution Camera (HRC) of Chandra. They estimate that the $\mathrm{X}$-ray luminosity of this component is $\sim 10^{34} \mathrm{ergs} \mathrm{s}^{-1}$, whereas the nonthermal nonpulsed X-ray luminosity from the Crab Nebula detected by $A S C A$ (Saito et al. 1998) is $\sim 10^{37} \mathrm{ergs} \mathrm{s}^{-1}$. The specific origin of the nonthermal nonpulsed component, not associated with the nebula, is not clear. Although it contaminates the emission of the nebula, it is small. Provided that this contribution is small for all the pulsars we study in this investigation, this component should not affect our analysis.

3. The nonthermal pulsed radiation component is generally believed to be produced in the pulsar magnetosphere. This follows from the fact that the motion of charged particles within the light cylinder is strongly affected by the magnetic field and 
the radiation of charged particles in the open field lines should be pulsed. This component could be produced in the vicinity of the polar cap as a result of the inverse Compton scattering of higher order generation pairs of particles on soft photons emitted by the neutron star (Zhang \& Harding 2000) or in the outer magnetosphere as a result of the synchrotron radiation of downward cascades from the outer gap electron/positron particles (Cheng \& Zhang 1999).

4. The thermal nonpulsed radiation from the pulsar surface can contribute to the soft X-ray bands, typically characterized by $k T \sim 0.1 \mathrm{keV}$ (Cheng \& Zhang 1999). In this paper, we have used the $A S C A$ data on pulsars, so this component will not contribute to the $2-10 \mathrm{keV} A S C A$ energy band.

5. The thermal pulsed radiation from the pulsar surface likely originates in a polar cap on the neutron star surface and can contribute to the hard X-ray band $(k T \sim 1 \mathrm{keV})$. In analyzing the pulsed emission from pulsars, we follow the model of Cheng \& Zhang (1999) in which the thermal pulsed X-ray emission is included in their model prediction.

Thus, the X-ray emission observed from pulsars in the $A S C A$ sample primarily consists of contributions from components 1 , 3 , and 5. We have assumed that component 2 is not important and that component 4 does not significantly contribute to the $2-10 \mathrm{keV}$ energy band. On the other hand, Becker \& Trümper (1997) have used ROSAT data to obtain a linear relation between the X-ray luminosity consisting of components 2 and 3 and the spin-down power. In the present paper, we concentrate on the X-ray properties in the energy band of ASCA (2$10 \mathrm{keV})$. The X-rays, then, consist of a thermal component from the polar cap, a nonthermal pulsed component from the magnetosphere, and a nonthermal, nonpulsed emission from the nebula. That is, we have explicitly assumed that the pulsar magnetosphere and the pulsar wind nebula are primarily responsible for the pulsed and nonthermal nonpulsed components, respectively.

Since the pulsed and nonpulsed emission have different origins, the relationship between the total X-ray luminosity and spin-down power is not expected, in general, to be represented by a single power law. In fact, the nonthermal pulsed and nonpulsed emission components are distinguished by different spectral signatures and conversion efficiencies. To determine their relative importance and their relationship to the spindown power, we have reexamined the pulsar data obtained in the $2-10 \mathrm{keV}$ energy band of $A S C A$, focusing on separating the nonpulsed and pulsed emission data. In the next section, we provide a simplified description of the emission from a pulsar wind nebula, based on earlier work by Chevalier (2000), in which the relativistic pulsar wind interacts with the interstellar medium (ISM). In $\S 3$, the X-ray properties of the pulsed and nonpulsed components are collected and the relation between their luminosities and spin-down power and photon spectral index are determined. With these results in hand, the pulsar wind nebula model is used to explain the occurrence of the tail-like emission morphology in Geminga and PSR B175724 in $\S 4$. Finally, we summarize and conclude in the last section.

\section{THEORETICAL FRAMEWORK}

A theoretical description of the interaction between a pulsar and its nebula, as applied to the Crab Nebula, was first outlined in a seminal paper by Rees \& Gunn (1974). In this model, the central pulsar generates a highly relativistic particledominated wind that passes through the medium in the supernova remnant, forming a shock front. The electrons and positrons in the shock are envisioned to be accelerated to a power-law energy distribution and to radiate synchrotron photons in the downstream region.

Since the magnetic field in the nebula derives from the pulsar, a magnetohydrodynamic (MHD) model, rather than a hydrodynamic model, of the pulsar wind nebula was required. Such a model, in the steady-state and spherically symmetric approximation, was developed by Kennel \& Coroniti (1984) for the Crab Nebula. A hot relativistic positronic plasma flow was assumed to be terminated by a strong MHD shock, decelerating the flow and producing a nonthermal distribution of electrons. The magnetization parameter, $\sigma$, introduced to describe the efficiency of the conversion of energy contained in the pulsar wind into synchrotron radiation, was represented by the ratio of the electromagnetic energy flux to the particle kinetic energy flux and defined as

$$
\sigma=\frac{B^{2}}{4 \pi n \gamma_{w} m c^{2}} .
$$

Here $n$ is the particle number density, $B$ is the magnetic field, $\gamma_{w}$ is the Lorentz factor of relativistic particles in the wind, $m$ is the particle mass, and $c$ is the speed of light. As pointed out by Kennel \& Coroniti (1984), the magnetization parameter must be small (for the Crab Nebula, $\sigma \sim 0.003$ ) in order for sufficient compression to occur in the shock for the transformation of the bulk flow energy to random particle motion and for the subsequent production of the observed synchrotron radiation. Since the magnetic energy density must dominate the particle energy density just inside the light cylinder, the wind nebula must have evolved from a high- $\sigma$ to a low- $\sigma$ state to produce the observed properties. The manner in which this transformation takes place is central to detailed pulsar wind models and is not understood in detail. Nevertheless, the low$\sigma$ model can account for the basic high-energy properties of the Crab, including the position of the optical wisps, the spectrum of the nebula, and the size of the nebula as a function of wavelength (van den Bergh \& Pritchet 1989).

In the present paper, we concentrate on the X-ray properties of the pulsar wind nebula, using a simple one-zone model similar to that developed by Chevalier (2000) for a description of the wind interaction with the surrounding medium in a variety of environments. Although the model does not provide a description of the spatial details associated with equatorial tori and polar jets observed in Chandra studies of young pulsar wind nebulae, it can explain the global X-ray properties of the nebula to a first approximation.

\subsection{One-Zone Model of X-Rays from Wind Nebulae}

In general, the energy in shock waves is stored in the magnetic field as well as in the proton (ion) and electron particle components. We assume that the fractional energy density of the magnetic field $\epsilon_{B}$ is $\sim 10^{-3}$ to $10^{-2}$ (Kennel \& Coroniti 1984). Provided that the spin-down power is eventually carried away by pulsar wind particles and assuming equipartition of energy between the electrons and protons, the fractional energy densities in the proton and electron components should satisfy $\epsilon_{p} \sim \epsilon_{e} \sim 0.5$. For a given $\epsilon_{B}$ in the shock, the magnetic field at the shock termination radius $R_{S}$ is estimated as $B=$ $\left(6 \epsilon_{B} \dot{E} / R_{s}^{2} c\right)^{1 / 2}$, where $\dot{E}=4 \pi I \dot{P} / P^{3}$ is the spin-down power of the pulsar. Here $P$ and $\dot{P}$ are the spin period and its derivative and $I$ is the moment of inertia of the neutron $\operatorname{star}\left(\sim 10^{45} \mathrm{~g} \mathrm{~cm}^{2}\right)$. 
At the shock front, the electrons attain a power-law distribution corresponding to $N(\gamma) \propto \gamma^{-p}$ for $\gamma_{m}<\gamma<\gamma_{\max }$, where $\gamma$ is the Lorentz factor and $\gamma_{m}=[(p-2) /(p-1)] \epsilon_{e} \gamma_{w}$. An estimate for $\gamma_{\max }$ can be obtained by equating the synchrotron cooling timescale to the electron acceleration timescale. The former timescale is given by $t_{\mathrm{syn}}=6 \pi m_{e} c / \sigma_{\mathrm{T}} \gamma B^{2}$ and the latter timescale is given by $t_{\mathrm{acc}}=\gamma m_{e} c / e B$, leading to $\gamma_{\max }=\left(6 \pi e / \sigma_{\mathrm{T}} B\right)^{1 / 2}$, where $m_{e}$ is the electron mass and $\sigma_{\mathrm{T}}$ is the Thomson cross section. The synchrotron power of an electron with Lorentz factor $\gamma$ is (Blumenthal \& Gould 1970)

$$
P(\gamma)=\frac{4}{3} \sigma_{\mathrm{T}} c \gamma^{2} \frac{B^{2}}{8 \pi}
$$

Following Chevalier (2000), the total rate of particles ejected by pulsars at Lorentz factor $\gamma$ is (Chevalier 2000)

$$
\dot{N}(\gamma)=(p-1) \gamma_{m}^{p-1}\left(\gamma_{w} m c^{2}\right)^{-1} \dot{E} \gamma^{-p} .
$$

The resulting total number of radiating particles becomes $N(\gamma) \sim \dot{N}(\gamma) t$, where $t$ is a characteristic timescale. For a young pulsar with a supernova remnant similar to the Crab, $t$ is identified with the age of the nebula, while for old pulsars or millisecond pulsars, $t$ is comparable to the flow timescale within the nebula. Thus, at the emitting frequency of an electron $\nu(\gamma)=$ $\gamma^{2} e B / 2 \pi m_{e} c$, the luminosity of the particle is about $P(\gamma) N(\gamma)$, which should be less than $\dot{E}$. The detailed properties of the X-ray nebula are a function of $\epsilon_{e}, \epsilon_{B}, R_{s}, \dot{E}$, and the two critical frequencies $\nu_{m}$ and $\nu_{c}$, where $\nu_{m}$ is the frequency radiated by the electrons with the Lorentz factor of $\gamma_{m}$ and

$$
\nu_{c}=18 \pi e m_{e} c / \sigma_{\mathrm{T}}^{2} t^{2} B^{3}
$$

is the electron synchrotron cooling frequency (see Chevalier 2000 and references therein).

The X-ray luminosity and the spectral index depend on the inequality between $\nu_{\mathrm{X}}$ and $\nu_{c}$. For typical values of $\epsilon_{e} \sim$ $0.5, \gamma_{w} \sim 10^{6}$, and $B \sim 10^{-4} \mathrm{G}, \nu_{m} \sim 10^{12} \mathrm{~Hz}$ is always less than $\nu_{\mathrm{X}}$. In this case, the X-ray luminosity from the total emission of particles per unit frequency can be calculated according to the synchrotron spectral profiles that have been presented in Figure 1 of Sari et al. (1998). For $\nu_{\mathrm{X}}>\nu_{c}$, the luminosity per unit frequency can be obtained following equations (7) and (8) of Sari et al. (1998),

$$
L_{\nu} \simeq\left(\nu_{m} / \nu_{c}\right)^{-1 / 2}\left(\nu / \nu_{m}\right)^{-p / 2} L_{\nu, \max }
$$

where $L_{\nu, \max }=N\left(\gamma_{m}\right) P\left(\gamma_{m}\right)$ and $\nu \sim 10^{18} \mathrm{~Hz}$. This expression corresponds to the fast-cooling regime, and is equivalent to equation (9) in Chevalier (2000), reflecting our use of the same form of particle number injection rate by a pulsar (see eq. [3] in this section). On the other hand, if $\nu_{\mathrm{X}}<\nu_{c}$ (denoted as slow cooling), the synchrotron $\mathrm{X}$-ray luminosity is estimated as (Sari et al. 1998)

$$
L_{\nu} \simeq\left(\nu / \nu_{m}\right)^{-(p-1) / 2} L_{\nu, \max }
$$

It can be seen that the X-ray luminosity in the fast-cooling regime is larger than that in the slow-cooling regime by a factor of $\left(\nu_{c} / \nu_{\mathrm{X}}\right)^{1 / 2}$ if $\nu_{\mathrm{X}}$ is in the slow-cooling regime. Since the range of $p$ due to shock acceleration lies between 2 and 3 (see Achterberg et al. 2001; Lemoine \& Pelletier 2003 and references therein), the photon index in the slow-cooling regime is expected to lie in the range 1.5-2 whereas the photon index in the fast-cooling regime is expected to be in the range $2-2.5$.

\subsection{Interactions between the Pulsar Wind and Surrounding Medium}

Within the theoretical framework, the energy of the electrons is converted to X-radiation solely at the shock termination radius. For pulsar motion that is subsonic, the determination of this radius is obtained via the balance between the wind ram pressure and the total magnetic and particle pressure within the nebula (Rees \& Gunn 1974). In this case, the wind bubble will be nearly centered about the position of the pulsar. Rees \& Gunn (1974) estimated $R_{s} \sim 3 \times 10^{17} \mathrm{~cm}$, which is consistent with the size of the inner X-ray ring of the Crab Nebula ( $\sim 0.1$ pc; see Kennel \& Coroniti 1984; Weisskopf et al. 2000). The termination shock picture is not necessarily restricted to slowly moving pulsars, since even for pulsars moving at several hundred $\mathrm{km} \mathrm{s}^{-1}$ the motion can be subsonic in, for example, regions where the gas has been heated to temperatures of $\sim 10^{8} \mathrm{~K}$ by a supernova shock. The radius of the termination shock can be estimated as

$$
R_{s} \simeq\left(\frac{\dot{E}}{B^{2} c}\right)^{1 / 2} \sim 6 \times 10^{14} \dot{E}_{34}^{1 / 2} B_{\mathrm{mG}}^{-1} \mathrm{~cm}
$$

where $\dot{E}_{34}$ is the pulsar spin-down power in units of $10^{34}$ ergs $\mathrm{s}^{-1}$ and $B_{\mathrm{mG}}$ is the magnetic field strength in the nebula in milligauss. With the observed values of the Crab pulsar and its nebula $\left(\dot{E}_{34}=5 \times 10^{4}\right.$ and $\left.B_{\mathrm{mG}}=0.5\right)$, consistency of the shock radius is easily achieved.

For supersonic motion, the nebula will form a bow shock morphology. In this case, the termination shock radius is given by the balance of the ram pressure between the wind particles and the medium at the head of the bow shock:

$$
\frac{\dot{E}}{4 \pi c R_{s}^{2}}=\frac{1}{2} \rho_{\mathrm{ISM}} v_{p}^{2}
$$

where $\rho_{\text {ISM }}$ is the density of the ISM and $v_{p}$ is the pulsar's proper motion velocity. The termination shock radius is given as

$$
R_{s} \simeq\left(\frac{\dot{E}}{2 \pi \rho_{\mathrm{ISM}} v_{p}^{2} c}\right)^{1 / 2} \sim 3 \times 10^{16} \dot{E}_{34}^{1 / 2} n_{1}^{-1 / 2} v_{p, 100}^{-1} \mathrm{~cm}
$$

where $n_{1}$ and $v_{p, 100}$ are the number density in the ISM in units of 1 particle $\mathrm{cm}^{-3}$ and the pulsar space velocity in units of $100 \mathrm{~km} \mathrm{~s}^{-1}$, respectively. As an example, Caraveo et al. (2003) recently discovered a bow shock structure coincident with the Geminga pulsar on the basis of observations obtained with XMM-Newton. An estimate of the termination radius for Geminga follows from its spin-down power, $\dot{E} \simeq 3.2 \times$ $10^{34} \mathrm{ergs} \mathrm{s}^{-1}$, a distance of $\sim 160 \mathrm{pc}$, and a proper motion velocity of $v_{p} \sim 120 \mathrm{~km} \mathrm{~s}^{-1}$ (Bignami \& Caraveo 1993), leading to a radius of $4 \times 10^{16} \mathrm{~cm}$, which is consistent with the observational constraint on Geminga's compact X-ray nebula.

The general features of the emission region surrounding rotation-powered pulsars in the Galaxy will be bracketed by 
these two wind nebula morphologies. Since the range in magnetic field strengths in the shock can vary from $\sim 10^{-5} \mathrm{G}$ to as high as $10^{-4} \mathrm{G}$ in the Vela Nebula and $5 \times 10^{-4} \mathrm{G}$ in the Crab Nebula for very young pulsars within supernova remnants, a range in the conversion efficiency of the spin-down energy into nebula luminosity is expected. The relation between the nebula $\mathrm{X}$-ray luminosity and the pulsar's spin-down power is deferred until $\S 3$.

\subsection{General Properties of the Wind Nebulae in X-Rays}

In the above, we have discussed the pulsar wind nebula models in different contexts within a one-zone approximation. The luminosities and spectral properties of the nebulae can be determined by equations (5) and (6) in the fast- and slowcooling regimes, respectively. In the fast-cooling case, the efficiency of converting spin-down power to nebula luminosity is higher than that in the slow-cooling case. Assuming that $\epsilon_{e} \sim 0.5, \epsilon_{B} \sim 0.01, \gamma_{w} \sim 10^{6}$, and $p \sim 2.2$ (see Bednarz \& Ostrowski 1998), the ratio of the X-ray luminosity $L_{\mathrm{X}} \sim \nu L_{\nu}$ to the spin-down power at $\nu \sim 10^{18} \mathrm{~Hz}$ can be estimated as $L_{\mathrm{X}} / \dot{E} \sim 10^{-2}$ to $10^{-1}$ for the fast-cooling case and $L_{\mathrm{X}} / \dot{E} \sim$ $10^{-4}$ to $10^{-3}$ for the slow-cooling case if $t \sim 10^{9} \mathrm{~s}$ and $B \sim$ $10^{-5} \mathrm{G}$. The spectral properties in these two regimes differ, since the photon index in X-rays is $\Gamma=(p+2) / 2$ and $(p+$ 1) $/ 2$ for fast and slow cooling, respectively. Since $p$ varies from 2 to $3, \Gamma \sim 1.5-2.0$ (slow cooling) and 2.0-2.5 (fast cooling). These are typical of the photon indices of observed X-ray nebulae (see Table 1 of Chevalier 2000).

As described in $\S 2.1$, the fast- and slow-cooling regimes are distinguished by the X-ray and cooling frequencies. Since the cooling frequency is dependent on the magnetic field and the flow timescale of the nebula, it is possible that different regions of the nebula may correspond to different cooling regimes. For example, the inner region of the nebula may correspond to the slow-cooling regime, whereas the fast cooling may be more appropriate in the outer region of the nebula. In this case, the power-law index of the X-ray spectrum will steepen by $\sim 0.5$ between the inner and outer regions. This may be consistent with the spectral results from the high spatial resolution observations of the nebulae surrounding PSR B1823-13 obtained with XMM-Newton (Gaensler et al. 2003b).

Because the spatial resolution of current X-ray detectors is limited, the emission from compact nebulae of size $\sim R_{S}$ may be contaminated by the nonthermal X-ray emission from the pulsar magnetosphere. However, the pulsar magnetospheric radiation is assumed to be pulsed, whereas the nebula contribution is nonpulsed. Therefore, the separation of the nonthermal X-ray emission into its pulsed and nonpulsed components is essential for determining their relative contributions.

\section{PULSED AND NONPULSED EMISSION}

Since the pulsed and nonpulsed emission components originate from different regions, their X-ray luminosities are not expected to exhibit a similar relationship to the spin-down power. For example, Wang \& Zhao (2004) recently found that the correlation of the observed pulsed X-ray luminosity with spin-down power is not well matched to the linear form found by Becker \& Trümper (1997) for the total luminosity in the ROSAT energy band $(0.1-2.4 \mathrm{keV}$; see Fig. 8 of Wang \& Zhao 2004). We note, however, that studies in this lower energy band are incomplete, since pulsars can emit a significant fraction of their luminosity at higher energies. To examine the X-ray luminosity-spin down power relation at higher energies (2$10 \mathrm{keV}$ ), Kawai et al. (1998) selected a sample of bright nebulae and found a relation in the form $L_{\mathrm{X}} \propto \dot{E}^{1.27 \pm 0.17}$. Since the individual components were not separately analyzed, the result was insufficient to establish a relation between the pulsed or nonpulsed emission with spin-down power.

\section{1. $L_{\mathrm{X}}-\dot{E}$ Relations from ASCA Data}

Over 50 rotation-powered pulsars have been detected in the X-ray band (Becker \& Aschenbach 2002), but only a fraction of them have been resolved with pulsed nonthermal components. Here we have selected $23 \mathrm{X}$-ray pulsars with both pulsed and nonpulsed emission measurements obtained from $A S C A$ (listed in Table 1). The pulsed and nonpulsed X-ray luminosities of these selected pulsars are taken directly from the cited references. This pulsar sample includes 19 normal pulsars and 4 millisecond pulsars whose observational data are taken from recent references (see Table 1). Since $A S C A$ does not have high spatial resolution, the X-ray luminosity of the pulsars in the $A S C A$ field is composed of emission from the pulsar's magnetosphere and compact pulsar wind nebula. For reference, the total pulsed plus nonpulsed X-ray luminosity in the ASCA energy range $(2-10 \mathrm{keV})$ is plotted versus spin-down power in Figure 1. A correlation is found that is consistent with the form $L_{\mathrm{X}} \propto \dot{E}^{1.5}$ found by Saito (1998), but the best-fit form of this correlation is found to be $L_{\mathrm{X}} \propto \dot{E}^{1.35 \pm 0.2}$. Here the error in the power-law exponent represents $\pm 1 \sigma$, corresponding to the scatter in the observed data points, which may reflect variations in $\epsilon_{e}, \epsilon_{B}$, and $\gamma_{w}$ and uncertainties in distance from pulsar to pulsar. Our best-fit power-law relation is consistent with the conclusion of Possenti et al. (2002), who used a sample of 39 pulsars observed mainly by ROSAT and data from ASCA, RXTE, BeppoSAX, Chandra, and XMM-Newton. Although Possenti et al. (2002) considered their result $\left(L_{\mathrm{X}} \propto \dot{E}^{1.34}\right)$ statistically unacceptable, our result is not subject to the uncertainties associated with the normalization of different satellite data to obtain the X-ray luminosity between 2 and $10 \mathrm{keV}$ (cf. Table 1 of Possenti et al. 2002), for which the extrapolation relied on the uncertain photon index in the ROSAT energy band.

The X-ray luminosity associated with the pulsed emission component is illustrated versus spin-down power in Figure 2. A correlation separate from the total luminosity is found that is inconsistent with either the form $L_{\mathrm{X}} \propto \dot{E}$ or $L_{\mathrm{X}} \propto \dot{E}^{3 / 2}$. The best-fitting function to the data is found to be $L_{\mathrm{X}, \mathrm{pul}} \simeq(1.0 \pm$ $0.6) \times 10^{-11} \dot{E}^{1.2 \pm 0.08}$, which significantly deviates from the $3 / 2$ power-law relation proposed by Saito (1998). Such a relation is consistent with the relation $L_{\mathrm{X}} \propto \dot{E}^{1.15}$, derived from the theoretical X-ray magnetospheric emission model of Cheng \& Zhang (1999); however, this latter result is not without uncertainties, since the inclination angle of the magnetic field with respect to the rotation axis and the viewing angle are not well determined. The observed conversion efficiencies are found to range from $\sim 10^{-5}$ to $9 \times 10^{-3}$, which is not in conflict with model predictions (Cheng et al. 1998; Cheng \& Zhang 1999).

A correlation is also found to exist between the nonpulsed X-ray luminosity and the spin-down power, as shown in Figure 3. The data points are consistent with the previous $A S C A$ relation of the type $L_{\mathrm{X}} \propto \dot{E}^{3 / 2}$, but the correlation is also consistent with $L_{\mathrm{X}, \mathrm{npul}} \propto \dot{E}^{1.4 \pm 0.1}$. On comparison with the results from Figure 1, this power-law relation is a consequence of the fact that the pulsar emission in the ASCA sample is dominated by the nonpulsed radiation component. The conversion efficiency for the nonpulsed component overlaps with that for the pulsed component, but extends to efficiencies as high as 0.1 . 
TABLE 1

Characteristics of Spin-powered Pulsars and Their X-Ray Luminosities Observed by ASCA

\begin{tabular}{|c|c|c|c|c|c|c|c|c|}
\hline $\begin{array}{c}\text { PSR } \\
(1)\end{array}$ & $\begin{array}{l}P \\
(\mathrm{~s}) \\
(2)\end{array}$ & $\begin{array}{c}\dot{P} \\
\left(\mathrm{~s} \mathrm{~s}^{-1}\right) \\
(3)\end{array}$ & $\begin{array}{c}d \\
(\mathrm{kpc}) \\
(4)\end{array}$ & $\begin{array}{c}\dot{E} \\
(5)\end{array}$ & $\begin{array}{c}L_{\mathrm{X}, \mathrm{tot}} \\
\left(\mathrm{ergs} \mathrm{s}^{-1}\right) \\
(6)\end{array}$ & $\begin{array}{c}L_{\mathrm{X}, \mathrm{pul}} \\
\left(\mathrm{ergs} \mathrm{s}^{-1}\right) \\
(7)\end{array}$ & $\begin{array}{c}L_{\mathrm{X}, \mathrm{npul}} \\
\left(\mathrm{ergs} \mathrm{s}^{-1}\right) \\
(8)\end{array}$ & $\begin{array}{c}\text { Reference } \\
\text { (9) }\end{array}$ \\
\hline $\mathrm{J} 0631+1036 \ldots \ldots \ldots \ldots \ldots$ & 0.288 & $1.0 \times 10^{-13}$ & 1.0 & $5.4 \times 10^{34}$ & $4.2 \times 10^{31}$ & $2.0 \times 10^{31}$ & $2.2 \times 10^{31}$ & 1 \\
\hline J1811-1926 _............... & 0.065 & $4.4 \times 10^{-14}$ & 5.0 & $7.0 \times 10^{36}$ & $1.6 \times 10^{34}$ & $1.9 \times 10^{33}$ & $1.4 \times 10^{34}$ & 2,3 \\
\hline B $0531+21 \ldots \ldots \ldots \ldots \ldots$ & 0.033 & $4.2 \times 10^{-13}$ & 2.0 & $4.5 \times 10^{38}$ & $1.1 \times 10^{37}$ & $6.8 \times 10^{35}$ & $1.0 \times 10^{37}$ & 4 \\
\hline В0833-45 .................. & 0.089 & $1.25 \times 10^{-13}$ & 0.3 & $6.9 \times 10^{36}$ & $1.9 \times 10^{33}$ & $1.5 \times 10^{32}$ & $1.8 \times 10^{33}$ & $4,5,6$ \\
\hline B0633+17.................. & 0.237 & $1.1 \times 10^{-14}$ & 0.16 & $3.2 \times 10^{34}$ & $8.2 \times 10^{29}$ & $4.6 \times 10^{29}$ & $3.6 \times 10^{29}$ & 7,8 \\
\hline B1706-44 .................. & 0.1025 & $9.3 \times 10^{-14}$ & 1.82 & $3.4 \times 10^{36}$ & $6.8 \times 10^{32}$ & $4.1 \times 10^{32}$ & $2.7 \times 10^{32}$ & 4 \\
\hline B1509-58 .................. & 0.150 & $1.54 \times 10^{-12}$ & 4.3 & $1.8 \times 10^{37}$ & $4.0 \times 10^{34}$ & $1.3 \times 10^{34}$ & $2.7 \times 10^{34}$ & 4 \\
\hline B1951+32................... & 0.0395 & $5.8 \times 10^{-15}$ & 2.5 & $3.7 \times 10^{36}$ & $6.2 \times 10^{33}$ & $6.2 \times 10^{32}$ & $5.6 \times 10^{33}$ & 4 \\
\hline В1046-58................... & 0.124 & $9.6 \times 10^{-14}$ & 2.98 & $2.0 \times 10^{36}$ & $9.5 \times 10^{32}$ & $5.5 \times 10^{32}$ & $4.5 \times 10^{32}$ & 4,9 \\
\hline B1929+10..................... & 0.227 & $1.16 \times 10^{-15}$ & 0.17 & $3.9 \times 10^{33}$ & $1.54 \times 10^{30}$ & $5.6 \times 10^{29}$ & $9.8 \times 10^{29}$ & 10 \\
\hline B0656+14 ...................... & 0.385 & $5.5 \times 10^{-14}$ & 0.76 & $3.8 \times 10^{34}$ & $1.7 \times 10^{31}$ & $1.0 \times 10^{31}$ & $7.0 \times 10^{30}$ & 4 \\
\hline В0540-69.................. & 0.05 & $4.8 \times 10^{-13}$ & 49.4 & $1.5 \times 10^{38}$ & $8.3 \times 10^{36}$ & $1.3 \times 10^{36}$ & $7.0 \times 10^{36}$ & 11 \\
\hline В0950+08.................. & 0.253 & $2.3 \times 10^{-16}$ & 0.12 & $5.6 \times 10^{32}$ & $4.6 \times 10^{29}$ & $1.6 \times 10^{29}$ & $3.0 \times 10^{29}$ & 10 \\
\hline B1610-50 ................. & 0.232 & $4.93 \times 10^{-13}$ & 7.26 & $1.6 \times 10^{36}$ & $9.6 \times 10^{33}$ & $3.0 \times 10^{33}$ & $6.6 \times 10^{33}$ & 9 \\
\hline B1055-52 …............... & 0.197 & $5.83 \times 10^{-15}$ & 1.53 & $3.0 \times 10^{34}$ & $2.7 \times 10^{30}$ & $2.0 \times 10^{30}$ & $7.0 \times 10^{29}$ & 12 \\
\hline B1853+01...................... & 0.267 & $5.4 \times 10^{-13}$ & 2.02 & $8.0 \times 10^{35}$ & $1.4 \times 10^{33}$ & $1.4 \times 10^{32}$ & $1.3 \times 10^{33}$ & 4 \\
\hline $\mathrm{J} 2229+6114 \ldots \ldots \ldots \ldots \ldots$ & 0.0516 & $7.8 \times 10^{-14}$ & 3.0 & $2.2 \times 10^{37}$ & $1.7 \times 10^{33}$ & $4.0 \times 10^{32}$ & $1.3 \times 10^{33}$ & 13 \\
\hline В0537-69................. & 0.016 & $5.13 \times 10^{-14}$ & 47 & $4.8 \times 10^{38}$ & $2 \times 10^{36}$ & $1.7 \times 10^{35}$ & $1.8 \times 10^{36}$ & 14,15 \\
\hline $\mathrm{J} 1846-0258 \ldots \ldots \ldots \ldots \ldots$ & 0.32 & $7.1 \times 10^{-12}$ & 19 & $1 \times 10^{37}$ & $1 \times 10^{36}$ & $4 \times 10^{34}$ & $1 \times 10^{36}$ & 16 \\
\hline B1937+21 .................. & 0.00156 & $1.05 \times 10^{-20}$ & 3.6 & $1.1 \times 10^{36}$ & $5.7 \times 10^{32}$ & $2.5 \times 10^{32}$ & $3.2 \times 10^{32}$ & 17 \\
\hline $\mathrm{J} 2124-3358 \ldots \ldots \ldots \ldots \ldots$ & 0.005 & $1.08 \times 10^{-20}$ & 0.25 & $3.5 \times 10^{33}$ & $4.8 \times 10^{29}$ & $1.6 \times 10^{29}$ & $3.2 \times 10^{29}$ & 18 \\
\hline B1821-24 ................. & 0.003 & $1.6 \times 10^{-18}$ & 5.1 & $2.2 \times 10^{36}$ & $6.5 \times 10^{33}$ & $9.4 \times 10^{32}$ & $5.5 \times 10^{33}$ & 19 \\
\hline 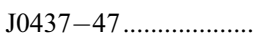 & 0.0058 & $2.0 \times 10^{-20}$ & 0.18 & $4.2 \times 10^{33}$ & $1.3 \times 10^{30}$ & $4.0 \times 10^{29}$ & $9.0 \times 10^{29}$ & 4 \\
\hline
\end{tabular}

Notes.-Col. (1): Pulsar name. Col. (2): Spin period. Col. (3): Period derivative. Col. (4): Distance of the pulsar from. Col. (5): The pulsar's spin-down power. Col. (6): Total X-ray luminosity observed by $A S C A(2-10 \mathrm{keV})$. Col. (7): Pulsed X-ray luminosity. Col. (8): Nonpulsed luminosity. Because the X-ray luminosities are all calibrated by $A S C A$, we can assume a $10 \%$ uncertainty for the total flux measurement and a 30\% fraction uncertainty, except for B1706-44, B1046-58, J1610-50, and J0437-47, which have a $50 \%$ uncertainty for the pulsed fraction.

References.-(1) Torii et al. 2001; (2) Torii et al. 1997; (3) Torii et al. 1999; (4) Saito et al. 1998; (5) Helfand et al. 2001; (6) Pavlov et al. 2001; (7) Halpern \& Wang 1997; (8) Cavaveo et al. 2003; (9) Pivovaroff et al. 2000; (10) Wang \& Halpern 1997; (11) Hirayama et al. 2002; (12) Shibata et al. 1997; (13) Halpern et al. 2001; (14) Marshall et al. 1998; (15) Wang \& Gotthelf 1998; (16) Gotthelf et al. 2000; (17) Takahashi et al. 2001; (18) Sakurai et al. 2001; (19) Saito et al. 1997.

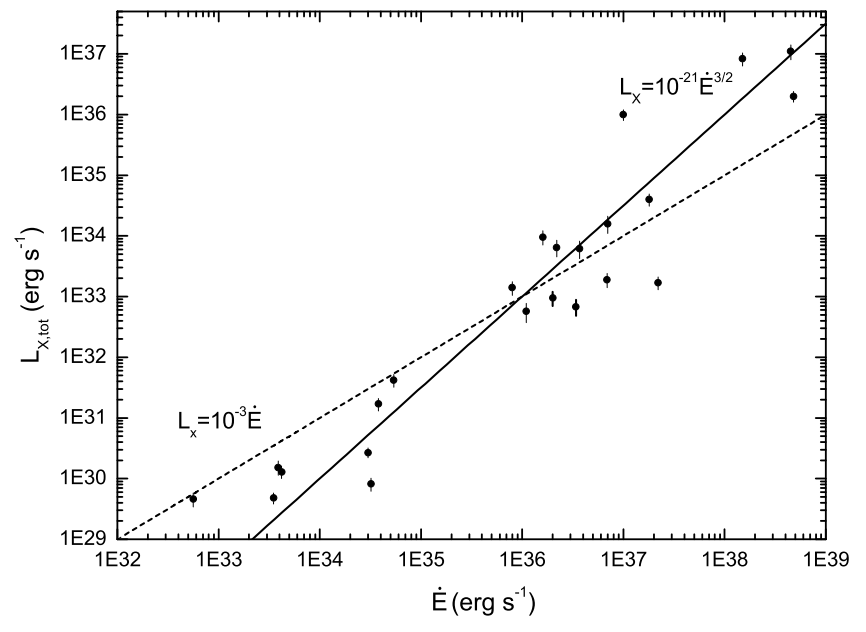

FIG. 1.-Total X-ray luminosity $(2-10 \mathrm{keV})$ from $A S C A$ observations vs. spin-down power of $23 \mathrm{X}$-ray pulsars. The solid line is $L_{\mathrm{X}}=10^{-21} \dot{E}^{3 / 2}$, as used by Saito (1998), and the dashed line represents $L_{\mathrm{X}}=10^{-3} \dot{E}$, as used by Becker \& Trümper (1997) to summarize ROSAT observations of central objects and pulsars. The best-fitting function is $L_{\mathrm{X}} \propto \dot{E}^{1.35 \pm 0.2}$, where the error in the power-law exponent represents $\pm 1 \sigma$, corresponding to the scatter in the observed data points.

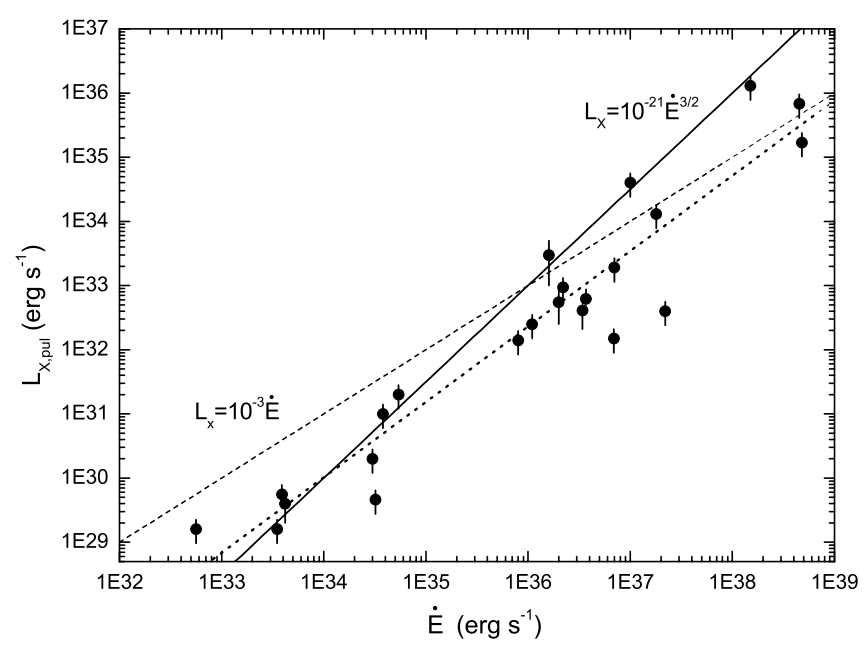

FIG. 2.-Pulsed X-ray luminosity $(2-10 \mathrm{keV})$ from $A S C A$ observations vs. spin-down power of $23 \mathrm{X}$-ray pulsars. The solid line is $L_{\mathrm{X}}=10^{-21} \dot{E}^{3 / 2}$ and the dashed line represents $L_{\mathrm{X}}=10^{-3} \dot{E}$. The relation between the pulsed component and the spin-down power cannot be described by both the two formulae. The best-fitting function is shown as the dotted line, $L_{\mathrm{X}}$, pul $=$ $10^{-11} \dot{E}^{1.2 \pm 0.08}$. 


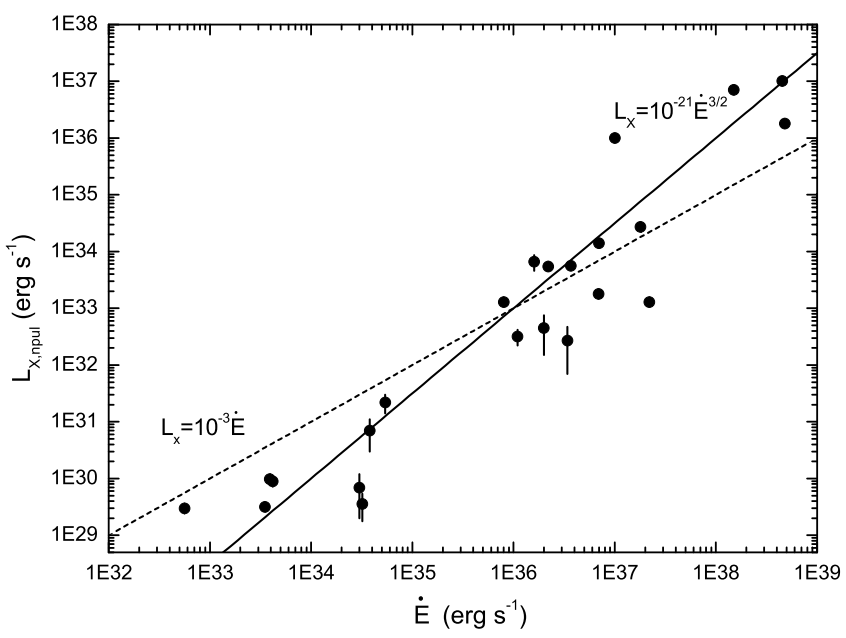

FIG. 3.-Nonpulsed X-ray luminosity (2-10 keV) from $A S C A$ observations vs. spin-down power of $23 \mathrm{X}$-ray pulsars. The solid line is $L_{\mathrm{X}}=10^{-21} \dot{E}^{3 / 2}$ and the dashed line represents $L_{\mathrm{X}}=10^{-3} \dot{E}$. The best-fitting function is $L_{\mathrm{X}} \propto \dot{E}^{1.4 \pm 0.1}$.

Generally, the X-ray luminosity of pulsar wind nebulae (Chevalier 2000) is a nonlinear function of the spin-down power. As can be seen from equation (9) of Chevalier (2000), the nebula's X-ray luminosity follows from

$$
L_{\mathrm{X}} \propto \epsilon_{e}^{p-1} \epsilon_{B}^{(p-2) / 4} \gamma_{w}^{p-2} R_{s}^{-(p-2) / 2} \dot{E}^{(p+2) / 4}
$$

Here $\epsilon_{e}$ and $\epsilon_{B}$ are assumed to be constant, but $R_{S} \propto \dot{E}^{1 / 2}$ from equations (7) and (9). Although the above equation corresponds to the fast-cooling regime, the dependence of $L_{\mathrm{X}}$ on $\dot{E}$ for the slow-cooling regime is unchanged because the ratio of the X-ray luminosities in these two regimes depends only on the cooling frequency $\nu_{c}$, which is independent of $\dot{E}$. We note that $\gamma_{w}$ also depends on the spin-down power, so the explicit dependence of $L_{\mathrm{X}}$ on $\dot{E}$ remains to be theoretically determined. To estimate this dependence we make use of the results of Ruderman (1981) and Arons (1983), who argued that large fluxes of protons (ions) could also be extracted from the neutron star and accelerated in the parallel electric field in the magnetosphere. The initial Poynting flux can be converted into particle thermal and kinetic energy well within the termination radius. Since both electrons and protons are basically accelerated by the low-frequency electromagnetic wave generated by the pulsar, they will be accelerated to the same relativistic speed, since they are bound by the strong electrostatic force. Hence, the Lorentz factor of the electrons and protons are the same, leading to the result that the protons may have carried away most of the spin-down power (Coroniti 1990). Thus, we can obtain a form for the spin-down power from

$$
\dot{E} \sim \dot{N} \gamma_{w} m_{p} c^{2}
$$

where $\dot{N}$ is the outflow current from the surface. This current should be on the order of the Goldreich-Julian current (Goldreich \& Julian 1969), given as $\dot{N} \simeq 1.35 \times 10^{30} B_{12} P^{-2}$ $\mathrm{s}^{-1}$. Since $\dot{E} \simeq 10^{31} B_{12}^{2} P^{-4}$ ergs s${ }^{-1}$, we find $\dot{N} \propto \dot{E}^{1 / 2}$, leading to $\gamma_{w} \propto \dot{E}^{1 / 2}$. Therefore, we obtain the relation $L_{\mathrm{X}} \propto \dot{E}^{p / 2}$, where $p$ generally varies between 2 and 3 . The relation deduced from the nonpulsed X-ray luminosity and spin-down power of observed pulsars may result from a relatively high electron energy index in the nebula.

\subsection{Statistical Properties of Pulsed and Nonpulsed Spectra}

Spectral observations of X-ray emission provide additional evidence in support of the pulsar wind nebula interpretation. In particular, the photon indices of both the pulsed and nonpulsed emission components, where determined, for X-ray pulsars are listed in Table 2. In this data compilation, the observational results are taken from the $A S C A$ data and also from the recent observations by BeppoSAX, Chandra, and XMM-Newton. In Figure 4, the photon index of the pulsed component is plotted versus the ratio of the isotropic pulsed X-ray luminosity to the pulsar spin-down power, $L_{\text {pul }} / \dot{E}$. The results reveal that the photon index of the pulsed emission component generally varies from 1.1 to 1.9 (except for PSR $0218+4232$ ). Such a range is consistent with the pulsed component originating in the pulsar's magnetosphere (Wang et al. 1998; Cheng et al. 1998). The values of $L_{\text {pul }} / \dot{E}$ vary over a wide range from $10^{-5}$ to $10^{-2}$ with no apparent correlation of the photon index with respect to efficiency. We note that the actual pulsed X-ray luminosities depend on the beaming of the pulse and therefore the conversion efficiency of spin-down power into pulsed X-ray luminosity is uncertain. The real efficiency may only scatter around an apparent efficiency, which is based on an assumed constant solid angle. Unless the beaming conspires with the photon index to produce a correlation, the rough distribution of the data presented in Figure 4 would not be expected to change. In summary, the efficiency and the photon index do not appear to show any correlation, which is consistent with the model predictions of Cheng \& Zhang (1999). On the other hand, Cheng \& Zhang (1999) suggest that the photon index sensitively depends on the local properties of the stellar magnetic field.

We also illustrate the relation between the efficiency of pulsar spin-down power to nonpulsed X-ray luminosity, $L_{\text {npul }} / \dot{E}$, and the photon index of the nonpulsed component in Figure 5. It can be seen that the photon index of the nonpulsed emission component shows two distinct regimes. In particular, there is a grouping of data points with $\Gamma_{\text {npul }} \lesssim 2$ for low efficiencies $\left(\lesssim 10^{-3}\right)$ and a separate grouping of data points with $\Gamma_{\text {npul }} \gtrsim$ 2 at higher efficiencies. This tendency may reflect a larger photon index in the fast-cooling regime in comparison to the slow-cooling regime, as discussed in $\S 2.3$. Specifically, we showed that the fast- and slow-cooling regimes are separated by $\left(\nu_{c} / \nu_{\mathrm{X}}\right)<1$ or $>1$, respectively, where $\nu_{c}$ is the cooling frequency and $\nu_{\mathrm{X}}$ is the observed $\mathrm{X}$-ray frequency. In the former regime, the photon indices are in the range from 2 to 2.5 and in the latter regime the photon indices range from 1.5 to 2 . Typically, the slow-cooling region has a lower efficiency than the fast-cooling region by a factor $\left(\nu_{c} / \nu_{\mathrm{X}}\right)^{1 / 2} \ll 1$. The actual efficiency of an individual pulsar depends on its corresponding parameters (e.g., $p, \gamma_{w}, B$, and $\epsilon_{e}$ ). Evidence in favor of such a relation is suggested by the difference between the Crab, which is in the fast-cooling regime with an efficiency of $\sim 0.03$, and Geminga, which is in the slow-cooling regime with an efficiency of $\sim 10^{-5}$.

\subsection{The Termination Radius and Nonpulsed Luminosity}

In Table 3, the termination radii derived from the images of some identified pulsar wind nebulae are listed. Although the determination of radius is imprecise, we have adopted two procedures for its estimation. For the nebulae that have ring structures (e.g., Crab Nebula), the termination radius is chosen 
TABLE 2

X-Ray Pulsed and Nonpulsed Component Properties of Spin-powered Pulsars

\begin{tabular}{|c|c|c|c|c|c|c|}
\hline $\begin{array}{c}\text { PSR } \\
\text { (1) }\end{array}$ & $\begin{array}{c}\dot{E} \\
\left(\underset{(2)}{\left.\operatorname{ergs~s}^{-1}\right)}\right. \\
(2)\end{array}$ & $\begin{array}{l}\eta_{\text {pul }} \\
\text { (3) }\end{array}$ & $\begin{array}{l}\eta_{\text {npul }} \\
\text { (4) }\end{array}$ & $\begin{array}{l}\Gamma_{\text {pul }} \\
(5)\end{array}$ & $\begin{array}{c}\Gamma_{\text {npul }} \\
\text { (6) }\end{array}$ & $\begin{array}{c}\text { Reference } \\
\text { (7) }\end{array}$ \\
\hline B1823-13....... & $2.8 \times 10^{36}$ & $3 \times 10^{-4}$ & $1.1 \times 10^{-3}$ & $1.6 \pm 0.1$ & $2.3 \pm 0.2$ & 1 \\
\hline J0537-6910 _........... & $4.8 \times 10^{38}$ & $3.6 \times 10^{-4}$ & $4 \times 10^{-3}$ & $1.6 \pm 0.2$ & $2.55 \pm 0.15$ & 2 \\
\hline B0540-69.................... & $1.5 \times 10^{38}$ & 0.01 & 0.05 & $1.83 \pm 0.13$ & $2.06 \pm 0.2$ & 3 \\
\hline B1509-58..................... & $1.8 \times 10^{37}$ & $7 \times 10^{-4}$ & $1.5 \times 10^{-3}$ & $1.36 \pm 0.02$ & $2.2 \pm 0.005$ & 4 \\
\hline J0218+4232 ................... & $2.5 \times 10^{35}$ & $4.8 \times 10^{-4}$ & $2 \times 10^{-4}$ & $0.61 \pm 0.32$ & $1.4 \pm 0.2$ & 5 \\
\hline J0631+1036 ................... & $5.4 \times 10^{34}$ & $5 \times 10^{-4}$ & $7 \times 10^{-4}$ & $2.3 \pm 0.5$ & $3.5 \pm 1.0$ & 6 \\
\hline $\mathrm{J} 1811-1926 \ldots \ldots \ldots \ldots \ldots$ & $7 \times 10^{36}$ & $3 \times 10^{-4}$ & $5 \times 10^{-3}$ & $1.65 \pm 0.26$ & $2.08 \pm 0.2$ & 7 \\
\hline $\mathrm{B} 0531+21 \ldots \ldots \ldots \ldots \ldots \ldots$ & $4.5 \times 10^{38}$ & $1.6 \times 10^{-3}$ & 0.03 & $1.6 \pm 0.2$ & $2.1 \pm 0.01$ & 8 \\
\hline 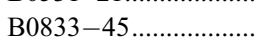 & $6.9 \times 10^{36}$ & $2 \times 10^{-5}$ & $3 \times 10^{-4}$ & $1.5 \pm 0.3$ & $1.67 \pm 0.04$ & 9 \\
\hline 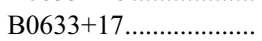 & $3.2 \times 10^{34}$ & $1.4 \times 10^{-5}$ & $1.1 \times 10^{-5}$ & $1.0 \pm 0.5$ & $1.6 \pm 0.2$ & 10,11 \\
\hline 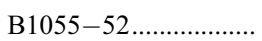 & $3 \times 10^{34}$ & $7 \times 10^{-5}$ & $2 \times 10^{-5}$ & $1.5 \pm 0.3$ & $1.9 \pm 0.2$ & 12 \\
\hline 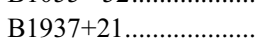 & $1.1 \times 10^{36}$ & $2 \times 10^{-4}$ & $3 \times 10^{-4}$ & $1.71 \pm 0.07$ & $0.8 \pm 0.6$ & 13 \\
\hline 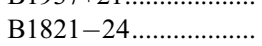 & $2.2 \times 10^{36}$ & $5 \times 10^{-4}$ & $3 \times 10^{-3}$ & $1.2 \pm 0.5$ & $1.89 \pm 0.22$ & 14 \\
\hline $\mathrm{J} 2229+6114 \ldots \ldots \ldots \ldots \ldots$ & $2.2 \times 10^{37}$ & $2 \times 10^{-5}$ & $10^{-4}$ & $1.51 \pm 0.14$ & $1.6 \pm 0.2$ & 15 \\
\hline J1105-6107 ................ & $2.5 \times 10^{36}$ & $6 \times 10^{-4}$ & $1.6 \times 10^{-3}$ & $1.8 \pm 0.4$ & $2.2 \pm 0.4$ & 16 \\
\hline B1706-44....................... & $3.4 \times 10^{36}$ & $1.4 \times 10^{-4}$ & $10^{-4}$ & $1.9 \pm 0.6$ & $1.7 \pm 0.5$ & 17 \\
\hline 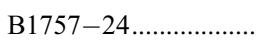 & $2.6 \times 10^{36}$ & $10^{-3}$ & $10^{-4}$ & $1.6 \pm 0.6$ & $1.0 \pm 0.6$ & 18 \\
\hline $\mathrm{J} 0205+6449 \ldots \ldots \ldots \ldots \ldots$ & $2.6 \times 10^{37}$ & $10^{-5}$ & $1.1 \times 10^{-3}$ & $1.1 \pm 0.4$ & $1.9 \pm 0.2$ & 19 \\
\hline 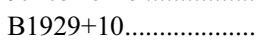 & $3.9 \times 10^{33}$ & $3 \times 10^{-4}$ & $6 \times 10^{-4}$ & $1.79 \pm 0.23$ & 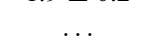 & 20 \\
\hline B0656+14 ....................... & $3.8 \times 10^{34}$ & $3 \times 10^{-4}$ & $2 \times 10^{-4}$ & $1.5 \pm 0.1$ & $\ldots$ & 21 \\
\hline $\mathrm{J} 2021+3651 \ldots \ldots \ldots \ldots \ldots$ & $3.6 \times 10^{36}$ & $3 \times 10^{-4}$ & $7 \times 10^{-4}$ & $1.5 \pm 0.3$ & $1.7 \pm 0.3$ & 22 \\
\hline J1747-2958 .................. & $2.5 \times 10^{36}$ & $10^{-3}$ & 0.02 & $1.8 \pm 0.1$ & $2.1 \pm 0.1$ & 23 \\
\hline $\mathrm{B} 1853+01 \ldots \ldots \ldots \ldots \ldots$ & $4.3 \times 10^{35}$ & $5 \times 10^{-4}$ & $1.5 \times 10^{-3}$ & $1.29 \pm 0.45$ & $2.2 \pm 0.2$ & 24 \\
\hline
\end{tabular}

Notes.-Col. (1): Pulsar name. Col. (2): Spin down power. Cols. (3) and (4): $\eta_{\text {pul }}=L_{\text {pul }} / \dot{E}$ and $\eta_{\text {npul }}=L_{\text {npul }} / \dot{E}$ are the X-ray conversion efficiencies of spin-down power to pulsed and nonpulsed X-ray luminosities, respectively. Cols. (5) and (6): $\Gamma_{\text {pul }}$ and $\Gamma_{\text {npul }}$ are the pulsed and nonpulsed photon indices, respectively.

RefERENCES.-(1) Gaensler et al. 2003b; (2) Marshall et al. 1998; (3) Kaaret et al. 2001; (4) Marsden et al. 1997; (5) Mineo et al. 2000; (6) Torii et al. 2001; (7) Torii et al. 1999; (8) Willingale et al. 2001; (9) Pavlov et al. 2001; (10) Halpern \& Wang 1997; (11) Cavaveo et al. 2003; (12) Shibata et al. 1997; (13) Takahashi et al. 2001; (14) Saito et al. 1997; (15) Halpern et al. 2001. (16) Gotthelf \& Kaspi 1998; (17) Finley et al. 1998; (18) Kaspi et al. 2001; (19) Murray et al. 2002; (20) Wang \& Halpern 1997; (21) Greiveldinger et al. 1996; (22) Hessels et al. 2004; (23) Gaensler et al. 2004; (24) Petre et al. 2002.

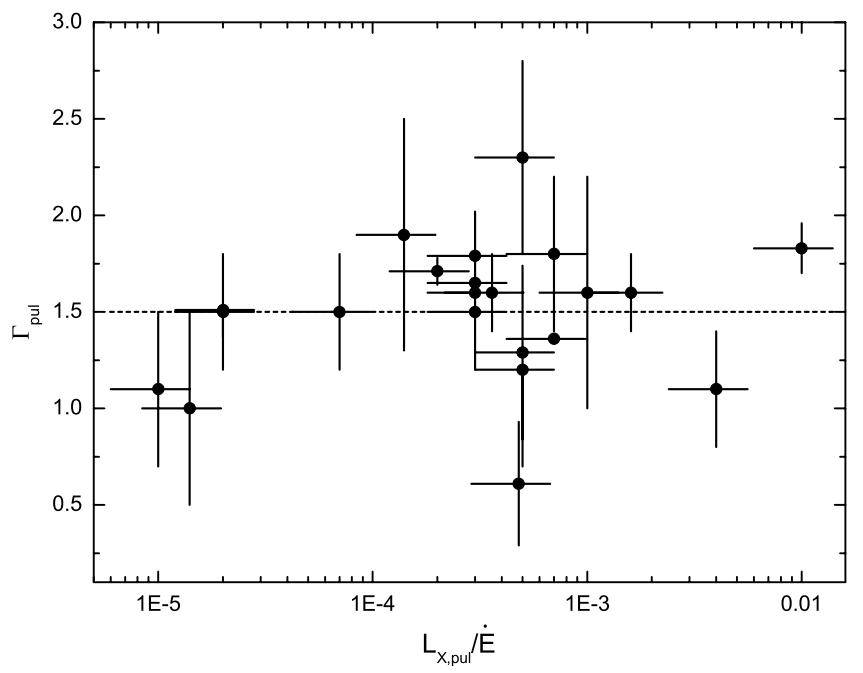

FIG. 4.-Photon index of the pulsed emission component of spin-powered X-ray pulsars vs. $\eta_{\text {pul }}=L_{\text {pul }} / \dot{E}$, the ratio of the isotropic pulsed X-ray luminosity to spin-down power.

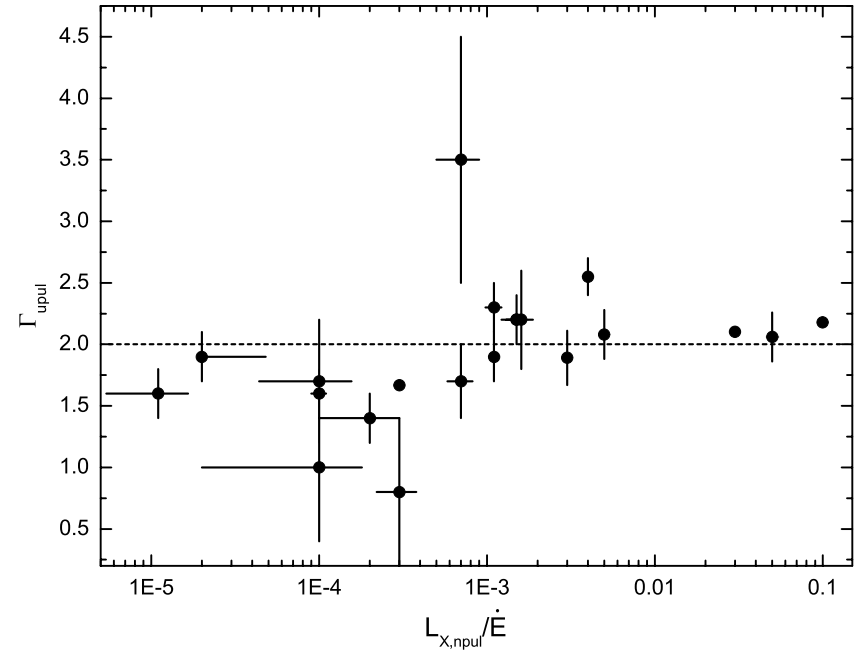

FIG. 5.-Photon index of the nonpulsed emission component of spinpowered X-ray pulsars vs. $\eta_{\text {npul }}=L_{\text {npul }} / \dot{E}$, the efficiency of spin-down power to nonpulsed X-ray luminosity. A dashed line corresponding to $\Gamma=2$, which approximately separates the fast-cooling regime from the slow-cooling regime, is shown for convenience. 
TABLE 3

X-Ray Properties of Pulsar Wind Nebulae

\begin{tabular}{|c|c|c|c|c|c|c|c|c|c|}
\hline $\begin{array}{c}\text { PSR } \\
\text { (1) }\end{array}$ & $\begin{array}{c}\dot{E} \\
\text { (2) }\end{array}$ & $\begin{array}{l}R_{s}^{\text {obs }} \\
(\mathrm{cm}) \\
(3)\end{array}$ & $\begin{array}{c}R_{s}^{\text {th }} \\
(\mathrm{cm}) \\
(4)\end{array}$ & $\begin{array}{c}L_{\text {upul }}^{\text {obs }} \\
\left(\mathrm{ergs} \mathrm{s}^{-1}\right) \\
(5)\end{array}$ & $\begin{array}{c}L_{\text {upul }}^{\text {th }} \\
\left(\mathrm{ergs} \mathrm{s}^{-1}\right) \\
(6)\end{array}$ & $\begin{array}{c}n \\
\left(\mathrm{~cm}^{-3}\right) \\
(7)\end{array}$ & $\begin{array}{c}v_{p} \\
\left(\mathrm{~km} \mathrm{~s}^{-1}\right) \\
(8)\end{array}$ & $\begin{array}{c}l^{\mathrm{a}} \\
(\mathrm{cm}) \\
(9)\end{array}$ & $\begin{array}{l}\text { Reference } \\
\text { (10) }\end{array}$ \\
\hline B1823-13 ……........... & $2.8 \times 10^{36}$ & $6 \times 10^{17}$ & $\ldots$ & $3 \times 10^{33}$ & $2 \times 10^{33}$ & 1 & $\ldots$ & $7 \times 10^{18}$ & 1 \\
\hline J0537-6910 _.................. & $4.8 \times 10^{38}$ & $4 \times 10^{17}$ & $\ldots$ & $1.8 \times 10^{36}$ & $3 \times 10^{36}$ & $\ldots$ & $\ldots$ & $\ldots$ & 2 \\
\hline B0540-69.................... & $1.5 \times 10^{38}$ & $3 \times 10^{17}$ & $\ldots$ & $7 \times 10^{36}$ & $4 \times 10^{36}$ & $\ldots$ & $\ldots$ & $\ldots$ & 3 \\
\hline J1811-1926 .................. & $7 \times 10^{36}$ & $2 \times 10^{17}$ & $\ldots$ & $1.4 \times 10^{34}$ & $10^{34}$ & $\ldots$ & $\ldots$ & $\ldots$ & 4 \\
\hline B $0531+21 \ldots \ldots \ldots \ldots \ldots$ & $4.5 \times 10^{38}$ & $4 \times 10^{17}$ & $3 \times 10^{17}$ & $10^{37}$ & $4 \times 10^{36}$ & 10 & 123 & $10^{18}$ & 5 \\
\hline В0833-45 .................. & $6.9 \times 10^{36}$ & $10^{17}$ & $2 \times 10^{17}$ & $1.8 \times 10^{33}$ & $10^{33}$ & 1 & 65 & $8 \times 10^{16}$ & 6 \\
\hline B0633+17....................... & $3.2 \times 10^{34}$ & $5 \times 10^{16}$ & $4 \times 10^{16}$ & $4 \times 10^{29}$ & $10^{30}$ & 1 & 120 & $4 \times 10^{17}$ & 7 \\
\hline $\mathrm{J} 2229+6114 \ldots \ldots \ldots \ldots \ldots$ & $2.2 \times 10^{37}$ & $4 \times 10^{17}$ & $\ldots$ & $1.3 \times 10^{33}$ & $3 \times 10^{33}$ & $\ldots$ & $\ldots$ & $\ldots$ & 8 \\
\hline $\mathrm{J} 1105-6107 \ldots \ldots \ldots \ldots \ldots$ & $2.5 \times 10^{36}$ & $4 \times 10^{16}$ & $\ldots$ & $4 \times 10^{33}$ & $3 \times 10^{33}$ & $\ldots$ & $\ldots$ & $\ldots$ & 9 \\
\hline В1706-44 ..................... & $3.4 \times 10^{36}$ & $3 \times 10^{17}$ & $\ldots$ & $3 \times 10^{32}$ & $4 \times 10^{32}$ & $\ldots$ & $\ldots$ & & 10 \\
\hline B1757-24_................. & $2.6 \times 10^{36}$ & $4 \times 10^{16}$ & $5 \times 10^{16}$ & $3 \times 10^{32}$ & $5 \times 10^{32}$ & 1 & 590 & $10^{18}$ & 11 \\
\hline J0205+6449 _.................. & $2.6 \times 10^{37}$ & $10^{17}$ & $\ldots$ & $3 \times 10^{34}$ & $3 \times 10^{34}$ & $\ldots$ & $\ldots$ & $\ldots$ & 12 \\
\hline B1957+20 & $10^{35}$ & $5 \times 10^{16}$ & $4 \times 10^{16}$ & $1.6 \times 10^{31}$ & $10^{31}$ & 1 & 220 & $4 \times 10^{17}$ & 13 \\
\hline $\mathrm{J} 2021+3651 \ldots \ldots \ldots \ldots \ldots$ & $3.6 \times 10^{36}$ & $8 \times 10^{17}$ & & $3 \times 10^{33}$ & $2 \times 10^{33}$ & $\ldots$ & $\ldots$ & $\ldots$ & 14 \\
\hline J1747-2958 ................. & $2.5 \times 10^{36}$ & $3 \times 10^{16}$ & $7 \times 10^{16}$ & $5 \times 10^{34}$ & $10^{34}$ & 0.3 & 600 & $2 \times 10^{18}$ & 15,16 \\
\hline $\mathrm{J} 1124-5916 \ldots \ldots \ldots \ldots \ldots$ & $10^{37}$ & $10^{17}$ & $2 \times 10^{17}$ & $4 \times 10^{34}$ & $10^{34}$ & 0.5 & 450 & $6 \times 10^{17}$ & 17 \\
\hline B1853+01..................... & $4.3 \times 10^{35}$ & $10^{17}$ & $3 \times 10^{16}$ & $6 \times 10^{32}$ & $4 \times 10^{32}$ & 5 & 375 & $10^{18}$ & 18 \\
\hline J1930+1852 _................. & $2 \times 10^{36}$ & $10^{17}$ & $\ldots$ & $10^{33}$ & $7 \times 10^{32}$ & 1 & $\ldots$ & $10^{18}$ & 19 \\
\hline В0453-685 ................... & $10^{37}$ & $6 \times 10^{17}$ & $\ldots$ & $6 \times 10^{34}$ & $2 \times 10^{34}$ & 0.4 & $\ldots$ & $3 \times 10^{18}$ & 20 \\
\hline J0538+2817 .................... & $4 \times 10^{34}$ & $8 \times 10^{16}$ & $3 \times 10^{16}$ & $6 \times 10^{31}$ & $5 \times 10^{31}$ & 0.5 & 385 & $\ldots$ & 21 \\
\hline
\end{tabular}

Notes.-Col. (1): Pulsar name. Col. (2): Spin-down power. Cols. (3) and (4): $R_{s}^{\text {obs }}$ and $R_{s}^{\text {th }}$ are the observed and the predicted termination radius of the pulsar wind nebulae, respectively. Cols. (5) and (6): $L_{\text {upul }}^{\text {obs }}$ and $L_{\text {upul }}^{\text {th }}$ are the observed and theoretical X-ray luminosities of pulsar wind nebulae, respectively. Col. (7): Number density of the medium around the pulsar. Col. (8): Pulsar proper motion velocity. Col. (9): Length of the observed X-ray elongated structure.

${ }^{a}$ Seven fast-moving pulsars in the bow shock show the X-ray tail features. The $l$ of the Crab and Vela pulsars corresponds to the outflow scales of the nebulae. For B0453-685 and J1930+1852, $l$ shows the scales of the elongated structure.

References.- (1) Gaensler et al. 2003b; (2) Marshall et al. 1998; (3) Kaaret et al. 2001; (4) Reynonds et al. 1994; (5) Weisskopf et al. 2000; (6) Pavlov et al. 2001; (7) Cavaveo et al. 2003; (8) Halpern et al. 2001; (9) Gotthelf \& Kaspi 1998; (10) Finley et al. 1998; (11) Kaspi et al. 2001; (12) Murray et al. 2002; (13) Stappers et al. 2003; (14) Hessels et al. 2004; (15) Gaensler et al. 2004; (16) Camilo et al. 2002; (17) Hughes et al. 2001; (18) Petre et al. 2002; (19) Lu et al. 2002; (20) Gaensler et al. 2003a; (21) Romani \& Ng 2003.

as the size of the inner ring. On the other hand, for the extended sources with a bright pointlike central sources the termination radius is taken to be the size corresponding to the scale in which $\sim 90 \%$ of the observed counts are included. For comparison, the theoretical termination radii for some nebulae are estimated according to equations (7) and (9) (for the Crab Nebula and other nebulae, respectively) as discussed in $\S 2.2$ and also listed in Table 3.

With the knowledge of the termination radius of the nebulae, the X-ray luminosity can be estimated assuming typical values for the parameters $\left(\gamma_{w}, p, \epsilon_{e}\right.$, and $\left.\epsilon_{B}\right)$. The observed and estimated X-ray luminosities of these compact nebulae are given in Table 3. For these estimates, we have taken $R_{S}$ as the observed values and assumed $\gamma_{w} \sim 10^{6}, p=2.2, \epsilon_{e} \sim 0.5$, and $\epsilon_{B} \sim 0.01$ for all pulsars. We find that the model luminosities and the observed luminosities are in rough agreement with each other to within a factor of 4 , which, taking into account the possible variations of these parameters amongst the pulsars, suggests that the simple one-zone model provides a reasonable approximation for the X-ray luminosity level.

\section{NORMAL PULSARS AND X-RAY TAILS}

The $\mathrm{X}$-ray-emitting region with a characteristic frequency $\left(\nu_{\mathrm{X}}=3 \gamma^{2} e B / 2 m_{e} c\right)$ may exhibit a tail-like spatial structure provided that the pulsar velocity exceeds that of the termination shock front and the nebula magnetic field is sufficiently low. In this case, the distance traversed by the pulsar within the synchrotron cooling timescale can be taken as a lower limit of the elongation length. Specifically, the synchro- tron cooling time in the X-ray band is $\tau_{c}=6 \pi m_{e} c / \gamma \sigma_{\mathrm{T}} B^{2} \sim$ $10^{8} B_{\mathrm{mG}}^{-3 / 2}\left(h \nu_{\mathrm{X}} / \mathrm{keV}\right)^{-1 / 2} \mathrm{~s}$, where $B_{\mathrm{mG}}$ is the magnetic field in the emission region in milligauss. Thus, the typical cooling time is $\sim 10^{11} \mathrm{~s}$ for $B_{\mathrm{mG}}=0.01$ and the length of the X-ray elongated feature is about $l \sim v_{p} \tau_{c} \sim 10^{18} \mathrm{~cm}$ for a pulsar moving at a velocity of $100 \mathrm{~km} \mathrm{~s}^{-1}$ with respect to the ISM.

The X-ray images of some pulsar wind nebulae, indeed, reveal extensive X-ray tails. In Table 3, several sources with $\mathrm{X}$-ray tail features are included. Seven cases are thought to be the consequence of a bow shock formed by a high-velocity pulsar (e.g., Geminga, PSR B1823-13, B1757-24, B195720, J1747-2958, J1124-5916, and B1853+01). The two pulsars J1930+1852 and B0453-685 exhibit elongated structures and may also be bow shock structures. On the other hand, the Crab and Vela Nebulae exhibit an outflow structure (X-ray jet).

\subsection{Geminga}

Geminga is one of the most studied pulsars because it is one of the very nearby pulsars and is the first pulsar detected in optical, X-ray, and gamma ray, but not in radio (Bignami \& Caraveo 1996) wavelengths. Based on ASCA observations, Saito et al. (1998) found that the nonpulsed X-ray luminosity was $\sim 3.6 \times 10^{29} \mathrm{ergs} \mathrm{s}^{-1}$ and the pulsed X-ray luminosity was $\sim 4.6 \times 10^{29} \mathrm{ergs} \mathrm{s}^{-1}$ in the $2-10 \mathrm{keV}$ band. On the basis of more recent XMM-Newton observations, Caraveo et al. (2003) has reported the detection of a power-law component $(\sim 7.7 \times$ $10^{29} \mathrm{ergs} \mathrm{s}^{-1}$ in the $2-8 \mathrm{keV}$ band), which consists of the pulsed as well as the nonpulsed component. The pulsed 
component in $2-6 \mathrm{keV}$ is $\sim 33.2 \% \pm 4.5 \%$, which is consistent with $A S C A$ observations. However, it is still not clear if the nonpulsed component originates from the pulsar or the nebula. The recent Chandra observations of the Geminga pulsar show an X-ray bow shock structure (Caraveo et al. 2003). The image of the field including the Geminga pulsar reveals a compact pointlike X-ray source of size $20^{\prime \prime}$ or $\left(6 \times 10^{16} \mathrm{~cm}\right)$ with two elongated tails extending to $2^{\prime}$ (Caraveo et al. 2003). Since the $\mathrm{X}$-ray tails have a nonthermal X-ray spectrum $(\Gamma \sim 1.6)$, these features may be produced by synchrotron emission in the bow shock between the pulsar wind and the ISM. The spin-down power of the Geminga pulsar is relatively low compared with other pulsars, with $\dot{E} \simeq 3.2 \times 10^{34} \mathrm{ergs} \mathrm{s}^{-1}$. Assuming a proper motion velocity of $v_{p} \sim 120 \mathrm{~km} \mathrm{~s}^{-1}$ (Bignami \& Caraveo 1993) and a number density of 1 particle $\mathrm{cm}^{-3}$ in the ISM, the termination shock radius of the pulsar wind nebula is $\sim 4 \times 10^{16} \mathrm{~cm}$. This scale is consistent with the observational constraint on the compact X-ray nebula for an assumed distance of $\sim 160 \mathrm{pc}$. The pointlike source has a X-ray luminosity of $1.2 \times 10^{30} \mathrm{ergs}$ $\mathrm{s}^{-1}$ in the energy range from 0.3 to $5 \mathrm{keV}$ and the total luminosity from the two tails is $\sim 6.5 \times 10^{28} \mathrm{ergs} \mathrm{s}^{-1}$ (Caraveo et al. 2003). The pulsed and nonpulsed emission of the pointlike source was not resolved in the XMM-Newton observations, but the results from $\S 3$ (see Table 1) reveal that the nonpulsed luminosity of Geminga is $3.6 \times 10^{29} \mathrm{ergs} \mathrm{s}^{-1}$. Interpreting this luminosity as arising from the compact X-ray nebula, we find that $L_{\text {neb, } \mathrm{X}} / \dot{E} \sim 10^{-5}$, which is low compared to other pulsar wind nebula. We suggest that the nebula is in the slow-cooling regime where the photon spectral index $\Gamma=(p+1) / 2$. The observed photon index of the tail is 1.6 , corresponding to $p=2.2$, and its observed length $\left(\sim 4 \times 10^{17} \mathrm{~cm}\right)$ suggests that the magnetic field of the surrounding medium is weak $(B \sim$ $1.5 \times 10^{-5} \mathrm{G}$; see also Caraveo et al. 2003). Thus, the inferred fractional magnetic energy density is $\epsilon_{B} \sim 10^{-3}$. Assuming a fractional electron energy density of $\epsilon_{e} \sim 0.5$ yields a nebula luminosity of $\sim 10^{30} \mathrm{ergs} \mathrm{s}^{-1}$ in the $2-10 \mathrm{keV}$ band, which modestly overestimates the contributions from the compact nebula and X-ray tails by a factor of $\sim 2.5$.

\subsection{PSR B1757-24}

Another example in which the model can be compared with observational data is the X-ray tail associated with PSR B1757-24, observed by Kaspi et al. (2001) with Chandra. PSR B1757-24 is a $124 \mathrm{~ms}$ radio pulsar discovered near the supernova remnant (SNR) G5.4-1.2 (Manchester et al. 1985), with a spin-down power $\dot{E} \sim 2.6 \times 10^{36} \mathrm{ergs} \mathrm{s}^{-1}$ (Manchester et al. 1991). Assuming an association between the pulsar and G5.4-1.2 and that the pulsar's characteristic age is a good

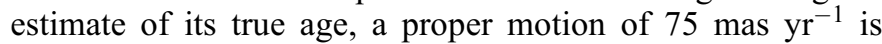
inferred. This corresponds to a transverse space velocity of $v_{p} \sim 1800 \mathrm{~km} \mathrm{~s}^{-1}$ for a distance of $5 \mathrm{kpc}$ (Frail et al. 1994). However, recent interferometric observations have failed to detect the implied proper motion (Gaensler \& Frail 2000), which suggests that the pulsar is older than its characteristic age or that the assumed pulsar birthplace is incorrect. Here we take the proper motion velocity of PSR B1757-24 to be $v_{p} \sim 590 \mathrm{~km} \mathrm{~s}^{-1}$ (Gaensler \& Frail 2000). The observed 2$10 \mathrm{keV}$ luminosity is $\sim 2 \times 10^{33} \mathrm{ergs} \mathrm{s}^{-1}$, corresponding to a conversion efficiency of $10^{-3}$, with the X-ray tail extending to $\sim 20^{\prime \prime}(0.5 \mathrm{pc}$ for the distance of $5 \mathrm{kpc})$. The observed photon index $\Gamma \sim 1.6$, yielding $p=2.2$ in the slow-cooling regime. Assuming an ISM number density $n \sim 1 \mathrm{~cm}^{-3}$, the termination shock radius is $R_{S} \sim 10^{17} \mathrm{~cm}$, with the known velocity $v_{p} \sim$ $590 \mathrm{~km} \mathrm{~s}^{-1}$. Taking the typical pulsar wind nebula parameters, $\epsilon_{e} \sim 0.5, \epsilon_{B} \sim 0.01$ (corresponding to $B \sim 20 \mu \mathrm{G}$ at $R_{s}$ ), and $\gamma_{w} \sim 10^{6}$, we find that the X-ray luminosity from $2-10 \mathrm{keV}$ is $\sim 3 \times 10^{33} \mathrm{ergs} \mathrm{s}^{-1}$ using the one-zone model. The predicted $\mathrm{X}$-ray tail length is given by $l \sim v_{p} t_{c} \sim 2 \times 10^{18} \mathrm{~cm}$. Both these estimates for the X-ray luminosity and tail length are in approximate accord with the observed values.

\section{SUMMARY}

The nonthermal nonpulsed X-ray emission of rotationpowered pulsars has been investigated in the context of emission from a pulsar wind nebula. We have confirmed that this emission can significantly contribute to the total X-ray emission, thereby increasing the X-ray emission above that produced in the pulsar magnetosphere. A re-examination of $A S C A$ data for pulsars separated into nonpulsed and pulsed emission components indicates that their nonthermal components, individually, are correlated with spin-down power. In particular, the dependence is steeper for the nonpulsed component, with $L_{\mathrm{X}} \propto \dot{E}^{1.4 \pm 0.1}$ as compared to $L_{\mathrm{X}} \propto \dot{E}^{1.2 \pm 0.08}$ for the pulsed component. Our results are similar to those discovered by Becker \& Trümper (1997), who found that the nonthermal (pulsed and nonpulsed) component satisfied the linear relation $L_{\mathrm{X}} \propto \dot{E}$, based on $R O S A T$ data in the $0.1-$ $2.4 \mathrm{keV}$ range. In this paper, we have used $A S C A$ data in the 2$10 \mathrm{keV}$ range, which includes the contribution of the additional thermal pulsed component, with $k T \sim 1 \mathrm{keV}$ from the polar cap (Cheng \& Zhang 1999). In this case, the dependence of the $\mathrm{X}$-ray luminosity on spin-down power is steeper. Because of the poor angular resolution of $A S C A$, the possible contribution of nonthermal nonpulsed emission from the magnetosphere is mixed in with that of the nebula.

Within the framework of a one-zone model for the pulsar wind developed by Chevalier (2000), the power-law relation between the nonpulsed luminosity and spin-down power provides an important diagnostic for the electron energy distribution in the shock that results from the interaction of the relativistic wind with the ISM. Specifically, $L_{\mathrm{X}} \propto \dot{E}^{p / 2}$, where $p$ is the power-law index of the electron energy distribution, suggesting that the index characterizing these pulsars is relatively high, $p \sim 2-3$. The observational results also show that the conversion efficiency of spin-down power into X-ray luminosity is a function of spin-down power, varying from $10^{-5}$ to 0.1 . The efficiencies in the lower (upper) end of the range can be understood in terms of emission in the slow (fast) cooling regime where the X-ray frequency is less (greater) than the electron synchrotron cooling frequency.

The spectra from pulsar wind nebulae are distinctly nonthermal, described by a photon power-law index, $\Gamma=(p+$ $2) / 2$ or $(p+1) / 2$ for the fast- or slow-cooling regimes, respectively. This implies a correlation between the photon index and the efficiency of conversion of spin-down power to nonpulsed X-ray luminosity, with steeper photon power-law indices associated with higher conversion efficiencies. Although the photon power-law indices inferred from the pulsars studied have large uncertainties, there are hints from the observed data that such a correlation exists. We note that the low values of $\Gamma$ and $L_{\mathrm{X}} / \dot{E}$ inferred for the extended emission observed from Geminga and B1757-24 are consistent with such an interpretation. Future observed determinations of the photon power-law indices could further verify this correlation, thereby providing an additional diagnostic tool for probing the pulsar and its environment.

Finally, the pulsar wind nebula interpretation may also apply to the spatially extended X-ray emission observed in binary 
systems containing a pulsar. For example, the extended X-ray emission associated with the millisecond pulsar B1957+20 (Stappers et al. 2003) may be interpreted within such a framework. The application of such models to recycled pulsars in the Galaxy will be the subject of a future investigation.
We are grateful to the anonymous referee for fruitful suggestions. This work is partially supported by the NSF through grant AST-0200876, by an RGC grant of the Hong Kong Government, and by the National Natural Science Foundation of China under grant 10273011.
Achterberg, A., Gallant, Y. A., Kirk, J. G., \& Guthmann, A. W. 2001, MNRAS, 328,393

Arons, J. 1983, in Electron-Positron Pairs in Astrophysics, ed. M. L. Burns, A. K. Harding, \& R. Ramaty (New York: AIP), 113

Becker, W., \& Aschenbach, B. 2002, in Proc. 270 WE-Heraeus Seminar on Neutron Stars, Pulsars, and Supernova Remnants, ed. W. Becker, H. Lesch, \& J. Trümper (Garching bei München: MPI), 64

Becker, W., \& Trümper, J. 1997, A\&A, 326, 682

Becker, W., Weisskopf, M. C., Tennant, A. F., Jessner, A., Dyks, J., Harding, A. K., \& Zhang, S. N. 2004, ApJ, in press

Bednarz, J., \& Ostrowski, M. 1998, Phys. Rev. Lett., 80, 3911

Bignami, G. F., \& Caraveo, P. A. 1993, Nature, 361, 704 1996, ARA\&A, 34, 331

Blumenthal, G., \& Gould, K. 1970, Rev. Mod. Phys., 42, 237

Camilo, F., Manchester, R. N., Gaensler, B. M., \& Lorimer, D. R. 2002, ApJ, 579, L25

Caraveo, P. A., Bignami, G. F., DeLuca, A., Mereghetti, S., Pellizzoni, A., Mignani, R., Tur, A., \& Becker, W. 2003, Science, 301, 1345

Cheng, K. S., Gil, J., \& Zhang, L. 1998, ApJ, 493, L35

Cheng, K. S., \& Zhang, L. 1999, ApJ, 515, 337

Chevalier, R. A. 2000, ApJ, 539, L45

Coroniti, F. V. 1990, ApJ, 349, 538

Finley, J. P., Srinivasan, R., Saito, Y., Hiriyama, M., Kamae, T., \& Yoshida, K. 1998, ApJ, 493, 884

Frail, D. A., Kassim, N. E., \& Weiler, K. W. 1994, AJ, 107, 1120

Gaensler, B. M., \& Frail, D. A. 2000, Nature, 406, 158

Gaensler, B. M., Hendrick, S. P., Reynolds, S. P., \& Borkowski, K. J. 2003a, ApJ, 594, L111

Gaensler, B. M., Schulz, N. S., Kaspi, V. M., Pivovaroff, M. J., \& Becker, W. E. 2003b, ApJ, 588, 441

Gaensler, B. M., van der Swaluw, E., Camilo, F., Kaspi, V. M., Baganoff, F. K., Yusef-Zadeh, F., \& Manchester, R. N. 2004, ApJ, in press

Goldreich, P., \& Julian, W. H. 1969, ApJ, 157, 869

Gotthelf, E. V., \& Kaspi, V. M. 1998, ApJ, 497, L29

Gotthelf, E. V., Vasisht, G., Boylan-Kolchin, M., \& Torii, K. 2000, ApJ, 542, L37

Greiveldinger, C., et al. 1996, ApJ, 465, L35

Halpern, J. P., Camilo, F., Gotthelf, E. V., Helfand, D. J., Kramer, M., Lyne, A. G., Leighly, K. M., \& Eracleous, M. 2001, ApJ, 552, L125

Halpern, J. P., \& Wang, F. Y.-H. 1997, ApJ, 477, 905

Helfand, D. J., Gotthelf, E. V., \& Halpern, J. P. 2001, ApJ, 556, 380

Hessels, J. W. T., Roberts, M. S. E., Ransom, S. M., Kaspi, V. M., Romani, R. W., Ng, C.-Y., Freire, P. C. C., \& Gaensler, B. M. 2004, ApJ, 612, 389

Hirayama, M., Nagase, F., Endo, T., Kawai, N., \& Itoh, M. 2002, MNRAS, 333,603

Hughes, J. P., Slane, P. O., Burrows, D. N., Garmire, G., Nousek, J. A., Olbert, C. M., \& Keohane, J. W. 2001, ApJ, 559, L153

Kaaret, P., et al. 2001, ApJ, 546, 1159

Kaspi, V. M., Gotthelf, E. V., Gaensler, B. M., \& Lyutikov, M. 2001, ApJ, 562, L163

Kawai, N., Tamura, K., \& Shibata, S. 1998, in IAU Symp. 188, The Hot Universe, ed. K. Koyama, S. Kitamoto, \& M. Itoh (Dordrecht: Kluwer), 265
Kennel, C. F., \& Coroniti, F. V. 1984, ApJ, 283, 694

Lemoine, M., \& Pelletier, G. 2003, ApJ, 589, L73

Lu, F. J., Wang, Q. D., Aschenbach, B., Durouchoux, P., \& Song, L. M. 2002, ApJ, 568, L49

Manchester, R. N., D’Amico, N., \& Tuohy, I. R. 1985, MNRAS, 212, 975

Manchester, R. N., Johnston, S., Kaspi, V. M., Lyne, A. G., \& D’Amico, N. 1991, MNRAS, 253, 7

Marsden, D., et al. 1997, ApJ, 491, L39

Marshall, F. E., Gotthelf, E. V., Zhang, W., Middleditch, J., \& Wang, Q. D. 1998, ApJ, 499, L179

Mineo, T. et al. 2000, A\&A, 355, 1053

Murray, S. S., Slane, P. O., Seward, F. D., Ransom, S. M., \& Gaensler, B. M. 2002, ApJ, 568, 226

Pavlov, G. G., Zavlin, V. E., Sanwal, D., Burwitz, V., \& Garmire, G. P. 2001, ApJ, 552, L129

Petre, R., Kuntz, K. D., \& Shelton, R. L. 2002, ApJ, 579, 404

Pivovaroff, M. J., Kaspi, V. M., \& Gotthelf, E. V. 2000, ApJ, 528, 436

Possenti, A., Cerutti, R., Colpi, M., \& Mereghetti, S. 2002, A\&A, 387, 993

Rees, M. J., \& Gunn, J. E. 1974, MNRAS, 167, 1

Reynolds, S. P., Lyutikov, M., Blandford, R. D., \& Seward, F. D. 1994, MNRAS, 271, L1

Romani, R. W., \& Ng, C.-Y. 2003, 585, L41

Ruderman, M. 1981, in IAU Symp. 95, Pulsars, ed. W. Sieber \& R. Wielebinski (Dordrecht: Reidel), 87

Saito, Y. 1998, Ph.D. Thesis, Univ. Tokyo

Saito, Y., Kawai, N., Kamae, T., \& Shibata, S. 1998, in Proc. Int. Conf. Neutron Stars and Pulsars: Thirty Years after Discovery, ed. N. Shibazaki et al. (Tokyo: Univ. Acad. Press), 295

Saito, Y., Kawai, N., Kamae, T., Shibata, S., Dotani, T., \& Kulkarni, S. R. 1997, ApJ, 477, L37

Sakurai, I., et al. 2001, PASJ, 53, 535

Sari, R., Piran, T., \& Narayan, R. 1998, ApJ, 497, L17

Seward, F. D., \& Wang, Z. 1988, ApJ, 332, 199

Shibata, S., et al. 1997, ApJ, 483, 843

Stappers, B. W., Gaensler, B. M., Kaspi, V. M., van der Klis, M., \& Lewin, W. H. G. 2003, Science, 299, 1372

Takahashi, M., et al. 2001, ApJ, 554, 316

Tennant, A. F., et al. 2001, ApJ, 554, L173

Torii, K., Tsunemi, H., Dotani, T., \& Mitsuda, K. 1997, ApJ, 489, L145

Torii, K., Tsunemi, H., Dotani, T., Mitsuda, K., Kawai, N., Kinugasa, K., Saito, Y., \& Shibata, S. 1999, ApJ, 523, L69

Torii, K., et al. 2001, ApJ, 551, L151

van den Bergh, S., \& Pritchet, C. J. 1989, ApJ, 338, L69

Wang, F. Y.-H., \& Halpern, J. P. 1997, ApJ, 482, L159

Wang, F. Y.-H., Ruderman, M., Halpern, J. P., \& Zhu, T. 1998, ApJ, 498, 373

Wang, Q. D., \& Gotthelf, E. V. 1998, ApJ, 494, 623

Wang, W., \& Zhao, Y. 2004, ApJ, 601, 1038

Weisskopf, M. C., et al. 2000, ApJ, 536, L81

Willingale, R., Aschenbach, B., Griffiths, R. G., Sembay, S., Warwick, R. S. Becker, W., Abbey, A. F., \& Bonnet-Bidaud, J.-M. 2001, A\&A, 365, L212 Zhang, B., \& Harding, A. K. 2000, ApJ, 532, 1150 